\title{
Calibrating urgency: triage decision-making in a pediatric emergency department
}

\author{
Vimla L. Patel · Lily A. Gutnik · Daniel R. Karlin · Martin Pusic
}

Received: 18 September 2006/ Accepted: 19 February 2007/Published online: 16 March 2007

(C) Springer Science+Business Media B.V. 2007

\begin{abstract}
Triage, the first step in the assessment of emergency department patients, occurs in a highly dynamic environment that functions under constraints of time, physical space, and patient needs that may exceed available resources. Through triage, patients are placed into one of a limited number of categories using a subset of diagnostic information. To facilitate this task and standardize the triage decision process, triage guidelines have been implemented. However, these protocols are interpreted differently by highly experienced (expert) nurses and less experienced (novice) nurses. This study investigates the process of triage; the factors that influence triage decision-making, and how the guidelines are used in the process. Using observations and semi-structured interviews of triage nurses, data was collected in the pediatric emergency department of a large Canadian teaching hospital. Results show that in emergency situations (1) triage decisions were often nonanalytic and based on intuition, particularly with increasing expertise, and (2) guidelines were used differently by nurses during the triage process. These results suggest that explicit guideline information becomes internalized and implicitly used in emergency triage practice as nurses gain experience. Implications of these results for nursing education and training, and guideline development for emergency care are discussed.
\end{abstract}

Keywords Triage - Decision-making - Emergency department - Nursing education · Clinical guidelines

\footnotetext{
V. L. Patel $(\bowtie) \cdot$ L. A. Gutnik · D. R. Karlin

Laboratory of Decision Making and Cognition, Department of Biomedical Informatics, Columbia University Medical Center, VC5 622 West 168th Street, New York, NY 10032, USA

e-mail: patel@dbmi.columbia.edu

M. Pusic

Division of Pediatric Emergency Medicine, Department of Pediatrics, Columbia University, New York, USA
} 


\section{Introduction}

Triage, as the gateway to patient care, plays a vital role in the emergency setting by categorizing and prioritizing patients efficiently according to their medical presentation (Williams 1996). Triage ensures that those patients with the greatest need for immediate care are able to receive treatment as quickly as possible, while patients with less immediate needs are treated as resources allow. If under-triaged, patients may be placed at a lower level of priority than they require leading to increased morbidity and mortality. Alternatively, over-triaged, minor cases also lead to increased morbidity of critical patients as the limited resources of the department become overburdened (Leprohon and Patel 1995). Though the ED is intended for emergency use, research has found that many patients present at the ED with non-urgent problems (Shesser et al. 1991; Buesching et al. 1985). Therefore, to effectively distribute limited resources (human, financial, technical, and institutional), the medical staff has to recognize who is most in need of care and must be able to reassess this as contextual factors change and additional patient information becomes available.

The goals of Triage, as set forth by the Canadian Association Of Emergency Physicians (CAEP), the National Emergency Nurses Affiliation of Canada (NENA), and L'association des médecins d'urgence du Québec (AMUQ) are: “(1) to rapidly identify patients with urgent, life threatening conditions (2) to determine the most appropriate treatment area for patients presenting to the ED (3) to decrease congestion in emergency treatment areas; to provide ongoing assessment of patients (4) to provide information to patients and families regarding services, expected care, and waiting times, and (5) to contribute information that helps to define departmental acuity', (Beveridge 1998).

As can be inferred from the goals outlined above, triage requires fine reasoning skills and decision-making based on sound clinical judgment. The nursing education community has agreed that the existing curricula are too focused on content and do not teach the most vital nursing skills of critical thinking, clinical reasoning, and decision-making (Candela et al. 2006). In fact, the National League for Nursing in the United States mandates that critical thinking skills must be included in nursing programs in order to gain accreditation (Simpson 2002). Yet the acquisition of this skill has scarcely been studied in the realm of nursing education (Baxter and Rideout 2006). Instead, these skills are acquired mainly through experience in the field (Oermann 1997; Candela et al. 2006). Research developments in cognitive psychology have been used as a foundation for Nursing Education (Roberts et al. 1993). Because it is imperative that nurses solve problems effectively, various techniques have been developed to assess a nurse's problem solving ability (e.g., Roberts et al. 1993). Regardless of this concerted effort to improve nursing efficiency, scientific evidence supports that this critical skill of problem solving has not been properly prioritized in the education curricula (Roberts et al. 1993). In response, nursing educators continue to pursue various new methodologies to build critical thinking skills (Hannah 2006). The process of nursing triage has a high density of decision-making such that it makes an excellent model for the study of critical thinking skills.

In this study, we investigate how triage decisions are made by nurses using a guidelinebased five-category system in a Canadian pediatric ED in 2000. Triage providers' characteristics are considered as they interact with the ambiguity of the protocol and influence decision-making. The results of this study have implications for the creation, implementation, and application of written triage guidelines as well as for nursing education and training. 
Triage decision-making

It is important to evaluate the significance of the triage guideline on the department and its patients (Washington et al. 2000; Beveridge et al. 1999) in terms of its effects on patient safety and the rapid and efficient delivery of patient care. To fully understand this impact, it is essential to examine the triage decision-making process with the use of these guidelines in the actual critical care setting (Washington et al. 2000; Zimmermann 2001; Travers 2002). The goal of the triage nurse is not to reach a diagnosis, but to focus on observing and quickly collecting as much relevant data as necessary and assign a category based on this information (Gerdtz and Bucknall 2001).

\section{Triage guidelines}

The Canadian Triage and Acuity Scale (CTAS) is the five-category triage guideline used in the Pediatric ED. Though the CTAS was designed for General ED use, there are pediatric practice considerations noted. For example, pediatric patients require more frequent reassessment than adults (Beveridge 1998). The original guidelines were proven to be reliable and valid (Beveridge et al. 1999). The guidelines are presented in text form consisting of eleven detailed pages of various aspects of the patient's condition corresponding to each of the triage categories as well as six pages of specific examples (Dong et al. 2005). Further revisions to the original guideline have been made since its initial implementation to expand the list of complaints and make the triage process more objective (Murray et al. 2004). The table below summarizes the five triage categories of CTAS. The categories are based on the extent of threat to life or limb due to the patient's condition. The time to be seen refers to the ideal maximum time allotted before patients should be seen by a physician as recommended by the Canadian Association of Emergency Physicians. Furthermore, it is important to note that a pediatric CTAS was established and implemented after we conducted this study (Table 1).

\section{Patient factors}

The decision-making process in triage is well defined in that specific parameters, such as vital signs, chief complaint and past medical history, are examined in a systematic manner and the results of this directed investigation lead to distinct decision options: one of five triage category assignments. The category assigned to each patient is directly dependent on the perceived severity of his/her illness.

Language barriers are a deterrent to the decision-making process. In the absence of interpreters or bilingual clinical staff, more cautious decision-making and increased resource use has been seen in ED studies (Hampers and McNulty 2002).

Table 1 The Canadian triage and assessment scale-the fivecategory triage protocol used in the pediatric ED based on Beveridge (1998)

\begin{tabular}{lll}
\hline Triage category & Definition & $\begin{array}{l}\text { Maximum time patient } \\
\text { can wait to be seen (in minutes) }\end{array}$ \\
\hline 1 & Resuscitation & Immediately \\
2 & Emergent & 15 \\
3 & Urgent & 30 \\
4 & Less-urgent & 60 \\
5 & Non-urgent & 120
\end{tabular}


Similarly, pediatric triage decision-making is complicated by the young age of the patients. Children may not cooperate during the triage process and there may be difficulty in obtaining vital signs and other diagnostic measurements (Mor and Waisman 2002).

\section{Contextual factors}

Despite the procedural nature of triage decision-making, the job of the triage nurse is complicated by the necessity of prioritizing each patient relative to other patients in the $\mathrm{ED}$, including patients within a triage category. The initial triage assignment is not static; it is subject to change if the condition of the patient degrades or improves (Gerdtz and Bucknall 2001). In the ED it is usually nurses with special training who gather the patients' medical information and make the initial triage decision. Although a nurse could consult an attending physician or a more senior nurse, this is not commonly done, so that the triage nurses are responsible for most of the triage decisions.

\section{Nurse factors}

A study of the decision-making process by nurses in the general emergency department found that nurses generated hypotheses based on both the information given by the patient and on single symptoms perceived as being characteristic of a diagnosis (Edwards 1994). The probabilities of these hypotheses were weighed against the nurse's knowledge and experience. The results showed that much of the decision-making was based on the nurse's experience. Marsden interviewed experienced nurses in an ophthalmologic ED and concluded that a significant amount of prior knowledge and experience was needed to quickly gather and consolidate the available information, "read between the lines," and arrive at the correct decision (Marsden 1998). In contrast, less experienced nurses felt that triage protocols were of more use to them. The nurses also rely on other factors such as medical, social, and contextual issues to assign a triage category. They were able to construe nonverbal cues to make the most efficient and accurate decision.

Nurses often use intuition to understand that something is wrong (Kenny 1994) rather than the guideline (Poole et al. 1993; Patel and Currie 2005). This result is found among other clinicians as well. Accordingly, a study found that more experienced physicians were more likely to break from protocols to achieve disposition of patient with possible cardiac chest pain than their less experienced counterparts (Pearson et al. 1994).

Lyneham investigated decision-making processes in emergency nurses and found that the use of the hypothetico-deductive model of clinical reasoning — using verbal, non-verbal and other sources of information, was also used by clinical nurse specialists in the emergency setting (Lyneham 1998). However, other studies show that experts do not use hypothetico-deductive process of reasoning (Patel et al. 2001c). It has further been shown that experienced nurses in medical-surgical units use heuristics rather than guidelines to rapidly and accurately form initial impressions of new patients (Simmons et al. 2003; Ferrario 2003). Similar effects take hold in triage, where it has been recognized that less experienced nurses look for textbook signs and symptoms to reach their diagnosis, whereas more experienced nurses base their decision on specific pieces of information provided by the patient as well as their past experiences (Cioffi 1998).

In a review of patient safety and cognition in nursing, Patel and Currie discuss the development of clinical expertise as well as differences between experts and novices. Contrary to popular assumption, performance is not linearly related to expertise. While 
performance is enhanced with the acquisition of expertise, the actual nature of this relationship is a U-shaped curve with an intermediate level of expertise exhibiting a decrease in performance (Patel and Groen 1986; Boshuizen and Schmidt 1992). This anomaly has been explained by the fact that experts have a well-organized knowledge base, novices lack the optimal knowledge base, and intermediates simply fail to make the necessary connections in their knowledge base. Another relevant model is the heuristic model, which suggests that clinical decision-making is driven by the rapid identification of specific models of disease or conditions. These models are improved as the clinician gains experience, thus subsequent decision-making relies more on exemplar and analogy use (Patel and Currie 2005). Benner has performed extensive investigations on the relationship between nursing experience and expertise and concluded that expertise is gained through experience via a five stage model of acquisition. Nonetheless, it does not strictly follow a U-Shape curve, but a linear curve with occasional lapses when new, unfamiliar situations arise (Benner 1984, 2004). Another study concluded that reasoning strategies among critical care nurses was consistent with those described in the realm of expertise, particularly in the vast use of heuristics (Fonteyn et al. 1991).

Based on existing literature as well as our data, we propose a model which illustrates the dynamics of triage decision-making. As viewed in Fig. 1, there are four major categories of factors that influence triage decisions: the nurse, the patient, guidelines, and the general status of the ED. Not only do these components independently influence the final triage decision, they often interact and influence the other components of the model. Our findings mainly center on the nurse factor of "experience" and how this interacts with guideline use.

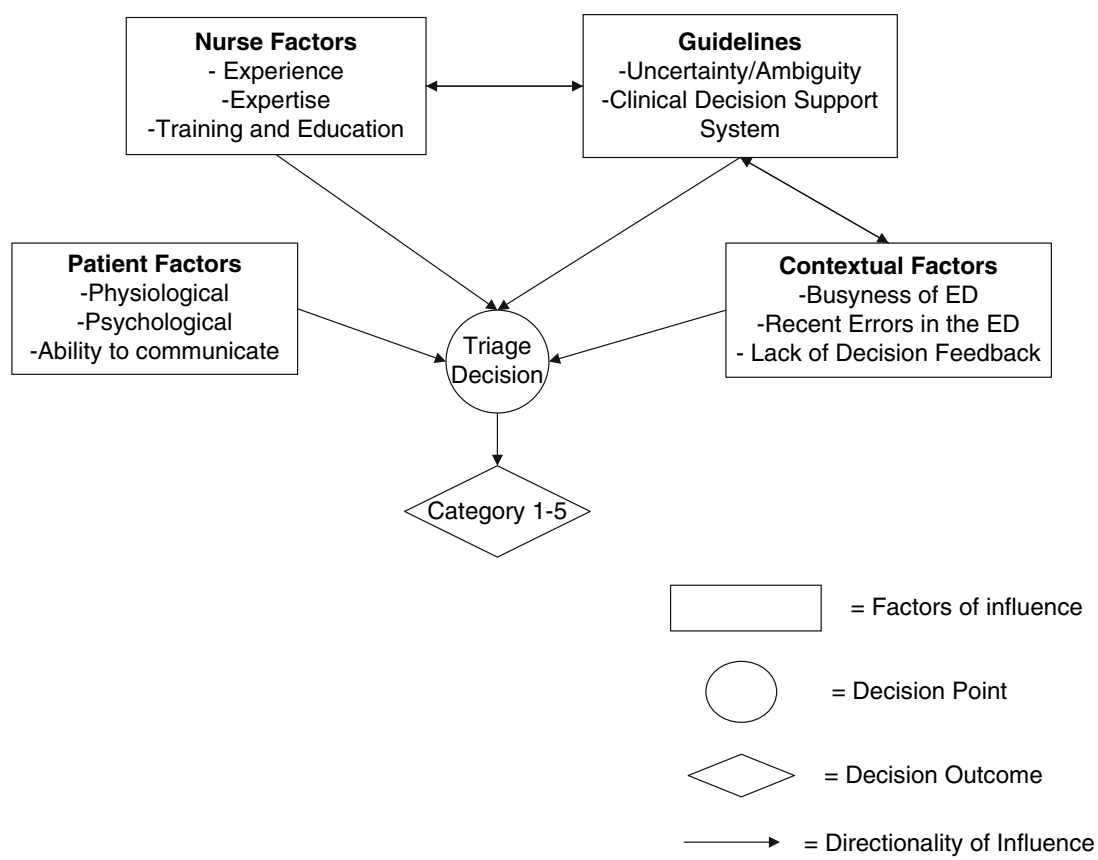

Fig. 1 Framework for factors involved in triage decision-making in pediatric ED 


\section{Methods and materials}

Description of the research environment

The study was conducted in 2000 in the pediatric emergency department of a large Canadian teaching hospital, which sees approximately 85,000 patients per year. After triage, the ED is composed of two main areas-the medical emergency areas and surgical emergency area. The larger part of the emergency department, the medical emergency area, treats medical conditions such as fevers, asthmatics, and children with less specific symptoms that do not generally require procedures. It is staffed by pediatricians and pediatric emergency medicine physicians. The Surgical emergency treats patients who have suffered injuries or illness which may require surgery. It is staffed by surgeons and pediatric emergency medicine physicians. The nursing staff does not generally specialize to one or other area.

Research design

\section{Data collection}

Strategies for data collection have been developed that allow the daily activity in workplace situations to be captured using ethnographic observational techniques, and semistructured interviews to gather data, thus facilitating a thorough understanding of staff members' day-to-day activities and behaviors (Patel et al. 1996; Patel et al. 2001a). In previous studies, these cognitive scientific methods have been found to be particularly effective in hospital environments (Patel et al. 2001a), such as in intensive care units, adult and pediatric emergency departments, and psychiatric emergency departments. The details of data collection are explained in the paragraphs below.

\section{Recruitment procedures}

Recruitment for participation in the study was done by members of the research study team verbally asking available triage nurses for their participation in the study. The nurse assignment schedule accounts for the variability among the times and days of data collection. Participation of the nurses is voluntary and they are assured that participation will not be a component of, or in any way influence, their status in the workplace.

\section{Emergency room observations}

Due to the fact that our objectives are to both outline and analyze how triage decisions are made in the highly complex environment of the ED, a naturalistic study was the preferred methodological approach. Based on the methods outlined by ethnography, the investigators were first stationed in the emergency department to observe triage activities and the triage environment, including the technology and the facilities involved. Over a period of time they familiarized themselves with the patterns of behavior in these settings. Subsequently, they sampled four different nurses on four different days (two mornings from 09:00 to 12:00, and two nights from 23:00 to 06:00 the next morning) over the course of three months (May-August 2000) in the ED. These specific times were selected because they represent times of high patient volume and were most convenient for our nurse collaborators. 
During these times the researchers followed the triage nurses when they triaged patients, and made notes on their observations. Data such as what information is used to make triage decisions, any references made to a text based guideline during triage, the flow of the patients throughout the $\mathrm{ED}$, and the use of technology during triage were documented.

\section{Semi-structured nurse interviews}

A representative sample of five triage nurses (four female and one male, the female to male ratio of the nursing staff) were interviewed by skilled members of the research team (including the first author). The limited availability of triage nurses for study participation accounts for the small sample size. All five nurses were informed of the potential risks of the research, especially regarding risks of loss of privacy, and consented to participate. Three nurses had more than 10 years working experience and the other two had fewer then ten. Each interview took about thirty minutes. The interviews were audio taped and later transcribed for analysis.

A semi-structured questionnaire comprised of nineteen questions addressing how triage decisions are made steered the interview. The questionnaire was developed after careful discussion by several researchers experienced in the area of decision-making who are also familiar with the administration of hospital emergency departments. These questions were divided into three categories: (1) factors directly affecting the process of triage, (2) the role of guidelines, and (3) additional characteristics of the ED that affect triage. For example, questions in the first category addressed how medical data gathered from the patient is used to assign a triage category. In the second category, the researcher was interested in learning about occasions when the guideline was intentionally or inadvertently disregarded. In the last category, questions such as the influence of technology on triage performance were posed.

\section{Analysis of nurse interviews}

Thematic coding, a qualitative coding scheme was developed. The scheme was developed via grounded theory approach, in which the primary investigator reviewed a subset of interview transcripts for emergent common themes using bottom up and inductive processing (Strauss and Corbin 1998). Responses were first divided into distinct themes such as the "concept of triage," "guidelines," "clinical decision-making," and "support" from a broad analysis of the protocols. Within the broad themes, clusters of related concepts were determined. For example, the category "decision-making'" was further partitioned into subtopics such as "basis for impression," "role of caregivers," and "major difficulties." Certain subtopics, namely those pertaining to the general theme of "decisionmaking," can be specified even further. For instance, "'general appearance,', "history,', "main complaint," "physical examination," and "vital signs" can be traced to the subcategory of "basis for impression", which itself is an offset of the broad topic, "decision-making.",

\section{Results}

The results are divided into two sections; the first describes the results based on observational data. The second section provides the results from interview analysis, documenting the nurse's perception of triage decision-making. 
Patient flow in the ED

From analysis of the notes generated during the observation period, we were able to piece together the patient flow through the emergency department. This generated workflow model was validated by the clinical staff. Figure 2 provides a conceptual model of patient and information flow through the ED. Upon entrance to the ED, the patient reported his chief complaint to the registration clerk. If the patient presented to the ED with a serious ailment, then he would bypass registration and immediately go to triage. After registration, patients are seen by a triage nurse. Patients triaged to category one, representing the highest level of urgency, would be immediately taken to the crash room and examined by a physician. Whereas categories $2-5$ are sent to the waiting room where they are subsequently called in for examination in order of urgency. At this stage the patients are monitored or re-triaged if necessary. A physician then sees the patient, evaluates their needs, and develops a plan of action to meet those needs.

\section{Triage procedure in the ED}

Nurses called the patients to triage according to their presenting complaint and the amount of time they have already been waiting. When the patient entered the triage room, the nurse usually began by recording the basic physical information, such as weight and temperature. Concurrently, the nurse asked the parents if the child was capable of verbal expression and questioned about the child's medical history. Subsequently, the nurse physically examined the patient and took the vital signs-pulse, temperature, blood pressure and respiratory rate. The nurse also performed a focussed physical examination related to the patient's complaint. For example, the nurse listened to the lungs if the child complained of a respiratory problem, and checked the nervous system if the child may have suffered a head injury. Sometimes additional diagnostic information was recorded to help with the decision. Blood oxygen saturation was taken to judge the extent of breathing difficulty, and a urine sample was taken if urinary tract infection was suspected. Finally, the nurse gathered supplemental information, such as the immunization record, allergies, and additional medical history. After all the information had been recorded on the triage form, the triage category was assigned and the patient was sent to the appropriate room. Category 1 patients bypassed the triage area entirely and initial assessment took place in a treatment room or trauma room while category 2 through 5 patients were either placed in an available exam room, or sat in the waiting room until an exam room was available. With the exception of category 1 patients, the time frame in which the patient was seen was primarily dependent on how busy the ED was at the time of arrival, and not solely on triage category. For example, if a category 3 patient came in at a time when there were category 1 or 2 patients in the ED, the category 3 patient would wait for the staff to stabilize the more serious patients. Conversely, if the ED was vacant, the category 3 patient would be seen almost immediately. Usually, category 4 and 5 patients were sent to the waiting room, category 2 and 3 patients were sent to an exam or observation room. Asthma patients triaged as category 2 or 3 were sent to the asthma treatment room for immediate inhalation therapy.

It was evident from the observations that an ED visit may cause worry or fear for the parents and family. To provide support and reassurance for the family, the nurses tried to listen to the parents and convey that their concerns were being taken seriously. Also, 


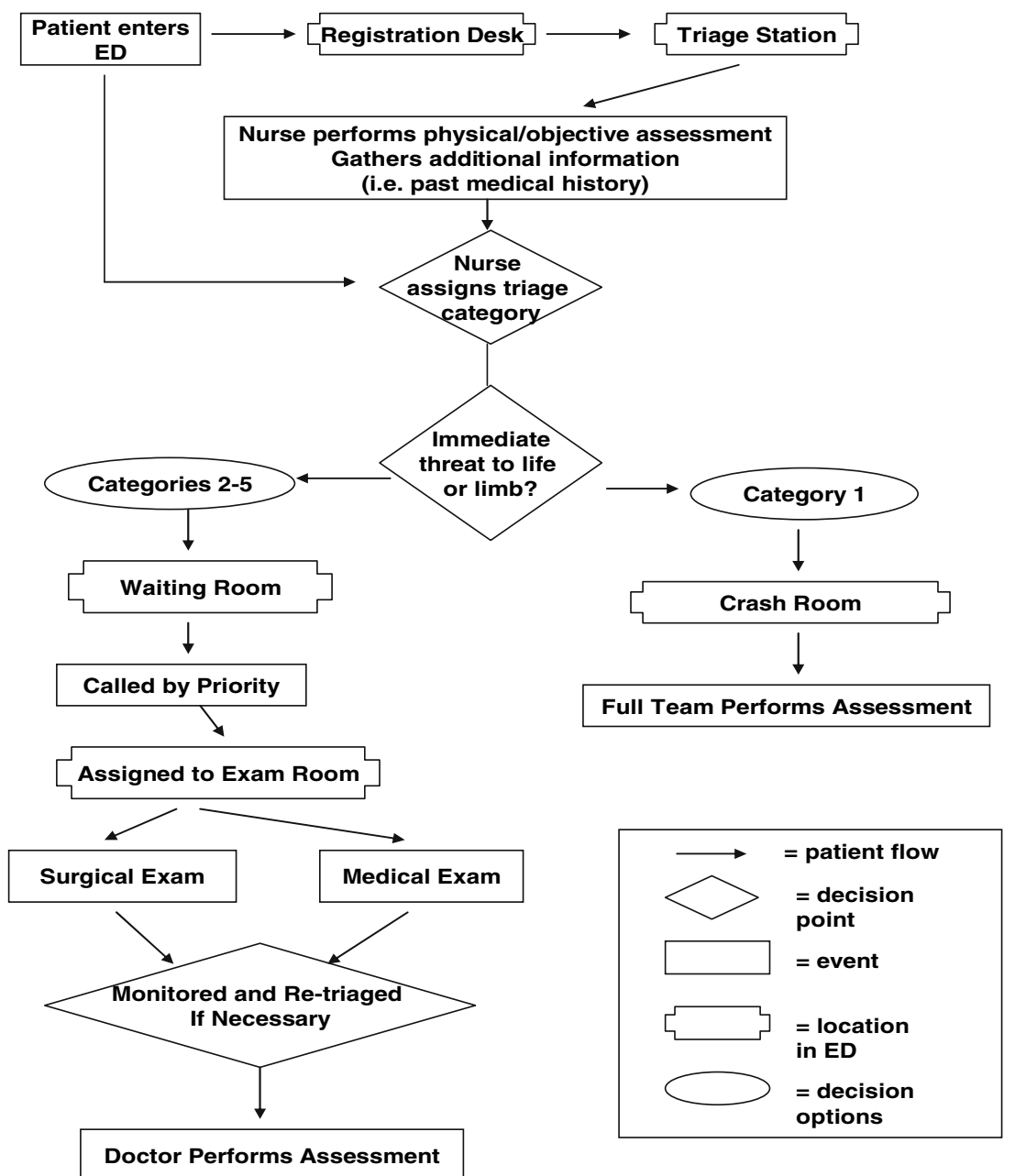

Fig. 2 Patient flow through the pediatric emergency department

parents were oriented as to the emergency department function, given an estimated waiting time, offered options if they did not wish to wait, and provided with updates on patient condition.

Nurses' perception of triage decision-making

\section{Factors directly involved in triage decisions}

The interview analysis yielded several broad categories of concepts salient to our participants, which the investigators further divided into narrower subtopics. For example, one of the subtopics of the category "clinical decision-making," was "basis for impression" which identified various distinctive traits evaluated by nurses in assigning the most 
accurate triage category. All of our subjects indicated that assessing the child's general appearance or "look" was usually the first and most helpful way to form an impression of the child's health status, especially for pre-verbal or inexpressive children. Interestingly, a difference in decision-making techniques was identified between experienced and inexperienced triage nurses. This was shown to be related to the level of experience as illustrated by one nurse, who responded "Really, with experience you do not really go by the guidelines, in the sense that it's self-evident.... You do it automatically.' Conversely, less experienced nurses, although they value their first impression as important, placed more emphasis on the chief complaint and medical history for their assessment. To illustrate, one less experienced nurse stated, "You have to look at everything. But the first thing is your ABCs and I, after that.... Your ABCs, vital signs and, just general appearance of so. You know changes of the colors, you know, the child looks dusky. Circulation.... Even, the heart rate, rhythm and sometimes the children are arrhythmic and this is also a concern."

Common practice to nurses of all experience levels were factors such as physical examination to make the appropriate triage decision when impression alone did not provide sufficient information. The low reliance on vital signs for making triage decisions is mirrored in other studies, one of which found that in greater then ninety percent of patients, nurses did not use the vital signs in making a decision (Cooper et al. 2002).

Another subtopic of the theme "clinical decision-making"' is the role of caregivers. A large proportion of the population served by the pediatric emergency department is preverbal, so that information that the clinician received about the patient's medical history and current state came from a third party or was observational information. In the case of somewhat older, yet not altogether verbal patients, the triage nurses and physician interacted verbally with the third party as well as the child. The older pediatric emergency patients were more involved in their own care. For the most part, however, a third party was involved with the medical care of the child until the age of 18 .

Our data show that, nurses carefully evaluate this third party information, as indicated by two of our five interviewees, and make judgments according to their own observations. This is due to the fact that information provided by third-parties is not always accurate or reliable for a number of reasons including: lack of health knowledge, misperceptions of the child's symptoms, and inadvertent exaggerations caused by anxiety. There are several methods employed by the nurses to judge the reliability of parents' description, such as comparing parents' words with those of the children's (if the child can speak), reported to be used by two of our interviewees, and rephrasing or rewording the same question to check consistency.

The results suggest that the quality and type of information provided by the child and their caregiver is a critical factor in triage decision-making, especially for inexperienced nurses.

\section{Use of guidelines in the ED}

"Guidelines", was a major theme that surfaced in the interviews. Nurses with varying levels of experience used the guidelines differently. The more experienced nurses usually generated a hypothesis based on their experience, intuition, and knowledge ( 2 out of 3 of our experienced nurses). If any discrepancy was perceived between their intuitions and the guideline, then they preferred to "go with their gut feelings" and disregard the guideline. On the contrary, both of the less experienced nurses from our sample revealed that they carefully followed the guideline most of the time. They relied on their intuition only if they 
were confident in their judgment, or under abnormal contextual conditions, such as a busy shift with a full waiting room. These results are consistent with other research in this area (e.g., Marsden 1998, Kenny 1994, Poole et al. 1993).

In the opinion of some nurses, the definitions of category 5 and category 4 are similar enough to be combined. They differ in that the new policy reduces the time within which a child with certain symptoms should be seen. Nurses felt that the new category definitions added substantial burden to their jobs without providing additional resources, such as staff or equipment. Thus, for those conditions that could be triaged as either category 4 or 5 , nurses usually would put the patients in category 5 .

The nurses reported that under some ambiguous situations, when they had difficulty differentiating between the appropriateness of two guideline categories, they would take precaution by assigning patients a higher priority category. Sometimes, they upgraded the patient's category because the parents were highly distraught or the nurse thought the patient would leave without being seen if they were left waiting too long. The nurses agreed that this sort of subjective assignment allows the nurses the flexibility to act outside the guidelines when they feel that modifying the priority queue might provide a specific benefit for a patient or a family.

The nurses report that the guideline is well defined and claim that it is followed most of the time. Yet, when the ED is very busy, 2 out of 5 of the nurses interviewed stated that it is hard to implement guideline recommendations due to factors such as the volume of patients, resource availability, and the parents' reaction to a child's illness. For example, because of a shortage of resources caused by an overload of patients, some patients could not be seen within the time prescribed by their triage category.

It is important to note that the triage guidelines are text-based. Though they are available in the triage room, the nurses do not have the time to continuously verify their triage decisions with the printed guidelines. This is consistent with guideline studies repeated elsewhere (Patel et al. 2001b). Additionally, some nurses have indicated that the presentation of the guidelines makes them difficult to use in a time pressured environment. Furthermore, about four months prior to the commencement of our study, the triage guidelines were revised from a four category scale to the five category CTAS scale. Many of the nurses indicated that they were still adjusting to this change.

In summary, our results suggest that guidelines are used much more frequently and rigorously by inexperienced nurses than experienced nurses, who have internalized the guidelines. Experienced nurses are more likely to use well-developed heuristics rather than external guidelines. However, under conditions of uncertainty, despite level of experience, guidelines are used for assistance and reassurance. Furthermore, text-based guidelines are difficult to use at the point of care.

\section{Contextual factors in triage decisions}

The remainder of the broad themes which surfaced from the interview analysis can be grouped together as non-clinical aspects which are integrated into triage decision-making separate from the nurse's own judgment and clinical reasoning skills. For example, when asked about interaction with the remainder of the medical staff, the nurses responded that most of the time, they consulted and collaborated with triage nurses or referred to the senior nurse when they were uncertain about a decision. Interaction with doctors during triage only occurs in very special circumstances. Nurses felt the most important factor that discouraged interaction between staff during the triage process was the time pressure, 
which made discussion and communication almost impossible, except when they interacted with the receptionists and coordinators for administrative purpose, i.e., arranging patients for entry when an exam room became available.

Largely as a result of the fast pace and high patient turnover; it was quite difficult for the triage nurses to obtain feedback regarding their decisions. Generally no further observation or evaluation was communicated back to the triage nurse after patients left the ED. Occasionally, because triage nurses also work in the other sections of the ED, they can get feedback indirectly for patients who are still in the department by reading the physician's diagnosis in the patient chart, as reported by 3 out of 5 of the interviewees. Feedback was obtained directly from the patients only if an individual is seen more than once by the same nurse in a short period of time. Additionally, in the case of poor outcomes, if there was a perception that the triage decision may have been a contributing factor, official feedback may be received in the form of incident reports or other administrative actions. Most nurses found that the feedback cycles focuses predominantly on the negative, with little or no official feedback received for making appropriate difficult decisions. These results are consistent with the results of other studies assessing the factors that triage nurses feel affect their performance (Cone and Murray 2002).

Another major theme of the interview results is the role of technology in triage. Computerized decision support tools were not installed in triage at the time of the study. Instead, the nurses specified that reference materials, such as textbooks, pamphlets, manuals, and guidelines were available for use. However, a new computer system called SIURGE was implemented in 1999 as an information source aid to facilitate the running of the ED. All the nurses had a high opinion of SIURGE, stating that it is a convenient and efficient method of acquiring patients' information, such as chief complaint, waiting time, location, triage category, etc., and it plays an important role in ED administration. However, they reported dissatisfaction with this technology in the fact that it cannot supply detailed medical information, such as medical history, general appearance, physical examination, and laboratory test results, which must be obtained from the patients' chart.

Language barriers were of great concern because difficulty in communicating with patients and parents impedes triage decision-making. If the parents could not speak or understand English or French (the two official languages in Quebec), an interpreter was called, usually arriving within thirty minutes of the request.

\section{Discussion}

Overall summary of results

Our results show that the triage nurse is responsible for examining the patients upon their presentation to the emergency department and classifying them into triage categories. This is expected to be in accordance with published guidelines. The components of pediatric triage are: general appearance, triage history, triage physical assessment, triage decision, health information and family support, and reassessment.

In addition to the level of experience and expertise of a nurse in assigning a triage category, circumstantial factors and hospital policies affect the decision-making process. These include the lack of feedback provided to the nurse for her triage decision, potential language barriers, the number of patients in the ED, and sources of decision support such as other medical personnel or reference material. 
Thinking and reasoning: the role of experience

Nurses with different levels of triage experience process information differently, thus employing different reasoning strategies to arrive at their decisions. Our finding that experienced nurses base their decision more on their "intuitive knowledge," which Benner and Tanner define as "understanding without a rational basis, based on background understanding and skilled observation" (Benner and Tanner 1987), was also congruent with Leprohon's (1995) study. Less experienced nurses rely more on the information from parents, given that less experienced nurses are not as confident in their own observations and need more information provided by external sources to confirm their decisions. In fact, it has been found that within a team of nurses, those with the most experience bring a sense of security and support to a group of inexperienced nurses (Andersson et al. 2006).

We noted a difference in the way that experienced and inexperienced nurses used the triage guidelines, with the inexperienced following it more closely. This could have implications for the inter-rater reliability of the CTAS. It should be noted that guidelines are of use only as a basic guide. At the time of an emergent situation, it is impossible to refer to the text-based guidelines for assistance or confirmation (Marsden 1998). However, since the time of our study some institutions have converted text based guidelines to tables, hoping that this will facilitate their use. Apart from the time pressure, shortages of staff and resources could also be reasons for disregarding the guideline.

Disregard for protocols and guidelines with increased clinical experience are also seen among physicians (Patel et al. 2001b). A systematic review assessing the relationship between clinical experience and health care outcomes indicates that physicians with more years of practice were consistently less likely to follow established guidelines on diagnostic and screening techniques. In contrast, younger physicians closely adhere to the guideline. This is also reflected in the finding showing a negative correlation between physician ages and following guideline standards (Choudhry et al. 2005).

Nevertheless, regardless of level of triage expertise, there are situations in which nurses choose to give the patient a high priority under ambiguous situations, such as a stomachache or headache. This tendency to err on the side of caution adds an additional burden to the ED resources and may result in providing inadequate attention to more urgent cases. On the contrary, it has also been suggested by one ED physician from our study that such triage tendencies are in fact adaptive and result in a better matching of resources to level of urgency. For example, he asserts that in handling an "overly" emotional patient, a good triage nurse will either (a) judge that this patient (or caregiver) is suffering more than "usual" and therefore expedite care or (b) assess whether the caregivers distress is actually appropriate (i.e., the parent is picking up on subtle cues that are not obvious to the nurse).

\section{Implications}

\section{Guideline improvement}

Our findings suggest that guidelines should be available in a format that is easily and quickly assessable at the point of care in the ER. The current guideline is text-based and divides disease into sixteen systems and five triage categories. It is slow to review and can be difficult to use, especially for the less-experienced nurses. An alternative format, either as clinical algorithms (Patel et al. 2001b) or clinical pathways which are easier to quickly 
scan during the course of an encounter (Katz 1999) is shown to be more valuable. However, these formats require explicit analysis of the logic structure and careful attention to the language used in guidelines (Patel et al. 2001a; Barak et al. 1998).

As expertise in a domain develops, people also develop problem solving short-cuts called heuristics. Similarly, as nurses become more experienced in triage, their decisions become automated and they refer less to text-based guidelines. For this reason, it is important that when training nurses for triage, these guidelines are well learned such that they are well incorporated into their knowledge structures. As medicine evolves and develops, guidelines are subject to change. Given this, we recommend that guidelines be appropriately updated and nurses receive the necessary continuing education as guideline modifications occur. Such recommendations may be difficult to implement as has been seen among older physicians. It has been suggested that one reason why more experienced physicians disregard modern guidelines is that these innovations are not within the physician's pre-existing knowledge framework (Choudhry et al. 2005). Therefore, guideline updates should always be designed with the user in mind (Buchman et al. 2006).

The use of health information technology is constantly evolving in healthcare settings. Triage support tools can be an essential addition to the triage system, and can also be used to train inexperienced nurses as well as experienced nurses on newly updated guidelines. For example, to alleviate some of the pressures in triage, a web-based triage tool called eTRIAGE, based on the five tier CTAS triage system, has been developed in Canada (Dong et al. 2005). This system allows the user to choose from a standard list of complaints. Based on these complaints, the system provides a template to further aid in deciding triage category. The nurse can disagree with the automated triage category choice, and choose an alternate category, but should note in the program reasons for disagreement.

\section{Triage nursing training and education}

Nurses are taught fundamentals in the stable, focused environment of the classroom. However, the classroom provides an unrealistic backdrop for the implementation of such important decision-making skills (Baxter and Rideout 2006). In reality, the ED is chaotic and unpredictable; thus, it is necessary to consider such an environment's effect on a nurse's decision-making skills (Patel et al. 2002). One suggestion to simulate the real world conditions in nursing education would be the use of case-based computer simulation. Such techniques have proven to produce successful outcomes of enhanced decision-making among midwifery students (Cioffi et al. 2005). Training based on simulation related to real patients in the context of a hectic, fast-paced ED may provide a more realistic learning environment. The nurse would have to attend to the needs of his or her "patient" to be triaged while bearing in mind the remaining "patients" waiting to be seen. Essentially, this simulation creates an ED setting where the nurse would have limited time, information, and resources to arrive at his or her decision. This suggestion is akin to the situated learning approach sometimes used in graduate medical education, where novice students learn from experts in the context of specific situations (Maudsley and Strivens 2000).

It should be recognized that a number of hospitals serve a large non-English speaking population. As shown in our study, language barriers are a major issue for triage nurses, especially in terms of comprehending the subtle nuances of critical messages. In effort to reduce such misunderstandings as well as to establish better clinician-patient communication for such hospitals, it would be wise to offer classes on basic foreign language skills for health professionals. 
Specific to pediatric emergency departments, most of the patient data is gathered from the parents. Though it is natural for parents to be highly concerned about their children, their concerns can inadvertently lead to somewhat exaggerated data. For this reason, nurses in pediatric triage training should be taught specific skills and techniques in information gathering when dealing with a third party, such as parents. This would allow the nurses to decipher which information is most accurate and pertinent. Efficient and effective data gathering skills for making optimal decisions are critical skills in the development of expertise in health professions.

Research has shown that experienced nurses make decisions more efficiently than less experienced nurses (e.g., Marsden 1998; Ferrario 2003). In triage, experienced nurses are able to quickly determine the most appropriate triage category whereas the inexperienced nurses take their time individually assessing every piece of data, not knowing what the optimal triage category will be until the very last step of their evaluation; an inherently less efficient process. Consistent with a review on physician guideline use (Choudhry et al. 2005) our key finding suggests that as clinicians become more experienced, they rely less on guidelines and focus more on individualizing rather than standardizing care for each patient. Thus, research that uses adherence to guidelines as a quality outcome measure may not always be accurate and reliable.

The experienced nurses spend less time focusing on the guidelines and rely on heuristics, which are developed through experience (Leprohon and Patel 1995). This is also evident among physicians (Patel et al. 1994; Higgins and Tully 2005). As expertise is developed, healthcare professionals develop more concrete and distinctive schemas (Higgins and Tully 2005). Unfortunately, these schemas or heuristics can be faulty (Patel et al. 2005). This finding only stresses the roles familiarity and practice play in a triage nurse's accuracy. Perhaps this concept should be incorporated in nursing training and education through nurse shadowing. If inexperienced nurses and nursing school students are required to spend considerable amounts of time shadowing experienced triage nurses, they will reiterate skills they have acquired and learn new ones through role modeling. However, this is a passive form of learning and may have to be supplemented by a more active form.

Another proposed method for bridging the performance gap between experienced and inexperienced nurses is the use of meta-cognitive strategy on when to consult a more experienced nurse during triage. The value of experience in education is exemplified by the experiential learning approach in medical education. This model proposes that the students engage in problem solving, practice this newly acquired knowledge in a reality, and then reflect on this experience. The reflection component of this model has been studied among physiotherapy students and been considered as one of the most vital and beneficial aspects of the learning process (Ward and Gracey 2006). Problem solving skills, the first portion of this model, have been viewed as the gateway to establishing good critical thinking skills (Maudsley and Strivens 2000). In fact, this was the rationale used by many U.S medical schools to adopt problem-based learning techniques, such as small-group case studies, into their curricula.

\section{Conclusions}

Due to the diversity of patients that pass through any ED, some standards of prioritization must be applied to make the process of receiving care productive and efficient. Such standards are identified by a multi-level triage acuity scale. But how are these protocols 
interpreted by different nurses and subsequently applied to individual patients? Our results along with previous studies indicate that experience is the most critical determinant of the nature of the use of guidelines. Differently structured knowledge and supporting reasoning strategies cause the less experienced nurse to follow the guidelines as though they were a text book-trying to perfectly match the patient's conditions to those described in the guideline. More experienced nurses have already internalized the guideline as part of their intuition and utilize prior experiences to gauge the acuity level for this patient. A problem for experienced nurses emerges when guidelines change. For example, a fifth category was only recently added to the previously defined four category acuity scale a few months before our study was conducted. Many nurses indicated that they saw little advantage in this change and found it difficult to distinguish between category 4 and 5 . The already existing guidelines are deeply rooted, thus making it difficult to break current patterns in thinking and reasoning.

Inaccurate decisions and systemic inefficiency of triage nurses, despite their degree of experience, can result in patients being under-triaged or over-triaged. Consequently, such faulty decisions are a source of potential harm due to the fact that patient's triage category assignment is a misrepresentation of their actual status. More naturalistic studies of triage decision-making in other health care settings would help gain additional understanding of the triage decision process. This could provide a basis for testing possible technological interventions to improve triage efficiency and accuracy.

Acknowledgments The research reported in this paper was supported by a grant from the Medical Research Council of Canada to Vimla Patel (MRC-MA-13439) and in part through the Department of Biomedical Informatics at Columbia University, New York. We would like to thank the participants in this study, and the nursing staff of the Emergency Department at McGill University, Montreal who made this study possible. We also wish to acknowledge David Kaufman and Laurie Barkun for their assistance with data collection and the development of the interview guide; and Eneida Mendoca and Elizabeth Nehemiah for their comments during the revision process.

\section{References}

Andersson, A. K., Omberg, M., \& Svedlund, M. (2006). Triage in the emergency department-a qualitative study of the factors which nurses consider when making decisions. Nursing in Critical Care, 11(3), 136-145.

Barak, N., Margolis, C. Z., \& Gottlieb, L. K. (1998). Text-to-algorithm conversion to facilitate comparison of competing clinical guidelines. Medical Decision Making, 18(3), 304-310.

Baxter P., \& Rideout E. (2006). Second-year baccalaureate nursing Students' decision making in the clinical setting. Journal of Nursing Education, 45(4), 121-127.

Benner, P. (1984). The Dreyfus model of skill acquisition applied to nursing. In P. Benner (Ed.), From novice to expert: Excellence and power in clinical nursing practice (pp. 13-38). Menlo Park: AddisonWesley.

Benner, P. (2004). Using the dreyfus model of skill acquisition to describe and interpret skill acquisition and clinical judgment in nursing practice and education. Bulletin of Science, Technology \& Society, 24(3), $188-199$.

Benner, P., \& Tanner, C. (1987). Clinical judgment: How expert nurses use intuition. American Journal of Nursing, 87(1), 23-31.

Beveridge R. (1998). CAEP issues. The Canadian triage and acuity scale: A new and critical element in health care reform. Canadian association of emergency physicians. Journal of Emergency Medicine, 16(3), 507-511.

Beveridge, R., Ducharme, J., Janes, L., Beaulieu, S., \& Walter S. (1999). Reliability of the Canadian emergency department triage and acuity scale: interrater agreement. Annals of Emergency Medicine, 34(2), 155-159. 
Boshuizen, H. P. A., \& Schmidt, H. G. (1992). On the role of biomedical knowledge in clinical reasoning by experts, intermediates, and novices. Cognitive Science, 16(2), 153-184.

Buchman, T. G., Patel, V. L., Dushoff, J., Ehrlich, P. R., Feldman, M., Levin, B., Miller, D. T., Rozin, P, Levin, S. A., \& Fitzpatrick, S. (2006). Enhancing the use of clinical guidelines: A social norms perspective. Journal of the American College of Surgeons, 201, 826-836.

Buesching, D. P., Jablonowski, A., Vesta, E., Dilts, W., Runge, C., Lund, J., \& Porter, R. (1985). Inappropriate emergency department visits. Annals of Emergency Medicine, 14(7), 672-676.

Candela, L., Dalley, K., \& Benzel-Lindley, J. (2006). A case for learning-centered curricula. Journal of Nursing Education, 45(2), 59-66.

Cioffi, J. (1998). Decision making by emergency nurses in triage assessments. Accident \& Emergency Nursing, 6(4), 184-191.

Cioffi, J., Purcal, N., \& Arundell, F. (2005). A pilot study to investigate the effect of a simulation strategy on the clinical decision making of midwifery students. Journal of Nursing Education, 44(3), 131-134.

Choudhry, N. K., Fletcher, R. H., \& Soumerai, S. B. (2005). Systematic review: The relationship between clinical experience and quality of health care. Annals of Internal Medicine, 142(4), 260-273.

Cone, K. J., \& Murray, R. (2002). Characteristics, insights, decision making, and preparation of ED triage nurses. Journal of Emergency Nursing, 28(5), 401-406.

Cooper, R. J., Schriger, D. L., Flaherty, H. L., Lin, E. J., \& Hubbell, K. A. (2002). Effect of vital signs on triage decisions. Annals of Emergency Medicine, 39(3), 223-232.

Dong, S. L., Bullard, M. J., Meurer, D. P., Colman, I., Blitz, S., Holroyd, B. R., \& Rowe, B. H. (2005). Emergency triage: Comparing a novel computer triage program with standard triage. Academic Emergency Medicine, 12(6), 502-507.

Edwards, B. (1994). Telephone triage: How experienced nurses reach decisions. Journal of Advanced Nursing, 19(4), 717-724.

Ferrario, C. G. (2003). Experienced and less-experienced nurses' diagnostic reasoning: implications for fostering students' critical thinking. International Journal of Nursing Terminologies \& Classifications, $14(2), 41-52$.

Fonteyn, M. E., Grobe, S. J., \& Kuipers, B. J. (1991). A descriptive analysis of expert critical care nurses' clinical reasoning. In E. J. S Hovenga, K. J. Hannah, K. A. McCormick, \& J. S. Ronald (Eds.), Nursing informatics '91 (pp. 765-768). Melbourne, Australia: Springer Verlag.

Gerdtz, M. F., \& Bucknall, T. K. (2001). Triage nurses' clinical decision making. An observational study of urgency assessment. Journal of Advanced Nursing, 35(4), 550-561.

Hampers, L. C., \& McNulty, J. E. (2002). Professional interpreters and bilingual physicians in a pediatric emergency department: Effect on resource utilization. Archives of Pediatrics \& Adolescent Medicine, 156(11), 1108-1113.

Hannah, V. (2006). The big picture: Learning to think like a nurse. Journal of Nursing Education, 45(6), 239-240.

Higgins, M. P., \& Tully, M. P. (2005). Hospital doctors and their schemas about appropriate prescribing. Medical Education, 39, 184-189.

Katz, D. A. (1999). Barriers between guidelines and improved patient care: An analysis of AHCPR's Unstable Angina Clinical Practice Guideline. Agency for Health Care Policy and Research. Health Services Research, 34(1 Pt 2), 377-389.

Kenny, C. (1994). Nursing intuition: can it be researched? British Journal of Nursing, 3(22), 1191-1195.

Leprohon, J., \& Patel, V. L. (1995). Decision-making strategies for telephone triage in emergency medical services. Medical Decision Making, 15(3), 240-253.

Lyneham, J. (1998). The process of decision-making by emergency nurses. Australian Journal of Advanced Nursing, 16(2), 7-14.

Marsden, J. (1998). Decision-making in A\&E by expert nurses. Nursing Times, 94(41), 62-65.

Maudsley G., \& Strivens J. (2000). Promoting professional knowledge, experiential learning and critical thinking for medical students. Medical Education, 34(7), 535-544.

Mor, M., \&Waisman (2002). Triage principles in multiple casualty situations involving children-The Israeli experience. Retrieved on January 14, 2006 from www. pem-database.org.

Murray, M. J., Bullard, M. J., \& Grafstein, E. (2004). Revisions to the Canadian emergency department triage and acuity scale implementation guidelines. Canadian Journal of Emergency Medicine, 6, 421427.

Oermann, M. H. (1997). Evaluating critical thinking in clinical practice. Nurse Educator, 22(5), 25-28.

Patel, V. L., Arocha, J. F., Diermeier, M., Greenes, R. A., \& Shortliffe, E. H. (2001a). Methods of cognitive analysis to support the design and evaluation of biomedical systems: The case of clinical practice guidelines. Journal of Biomedical Informatics, 34, 52-66. 
Patel, V. L., Arocha, J. F., Diemeier, M., How, J., \& Mottur-Pilson, C. (2001b). Cognitive psychological studies of representation and use of clinical practice guidelines. International Journal of Medical Informatics, 63(3), 147-168.

Patel, V. L., Arocha, J. F., \& Kaufman, D. R. (1994). Diagnostic reasoning and expertise. The Psychology of Learning and Motivation: Advances in Research and Theory, 31, 137-252.

Patel, V. L., Arocha, J., \& Lecissi, M. (2001c). Impact of undergraduate medical training on housestaff problem solving performance, implications for health education in problem-based curricula. Journal of Dental Education, 65(11), 1199-1218.

Patel, V. L., \& Currie, L. M. (2005). Clinical cognition and biomedical informatics: issues of patient safety. International Journal of Medical Informatics, 74(11-12), 869-885.

Patel, V. L., \& Groen, G. J. (1986) Knowledge-based solution strategies in medical reasoning. Cognitive Science, 10, 91-116.

Patel, V. L., Kaufman, D. R, \& Arocha, J. F. (2002). Emerging paradigms of cognition and medical decision making. Journal of Biomedical Informatics, 35, 52-75.

Patel, V. L., Kaufman, D. R. \& Magder, S. A. (1996). The acquisition of medical expertise in complex dynamic environments. In K. A. Ericsson (Ed.), The road to excellence: the acquisition of expert performance in the arts and sciences, sports, and games (p. xi, 369). Mahwah: Lawrence Erlbaum Associates.

Pearson, S. D., Goldman, L., Garcia, T. B., Cook, E. F., \& Lee, T. H. (1994). Physician response to a prediction rule for the triage of emergency department patients with chest pain. Journal of General Internal Medicine, 9(5), 241-247.

Poole, S. R., Schmitt, B. D., Carruth, T., Peterson-Smith, A., \& Slusarski, M. (1993). After-hours telephone coverage: the application of an area-wide telephone triage and advice system for pediatric practices. Pediatrics, 92(5), 670-679.

Roberts J. D., While A. E., \& Fitzpatrick, J. M. (1993). Problem solving in nursing practice: Application, process, skill acquisition and measurement. Journal of Advanced Nursing, 18(6), 886-891.

Shesser, R., Kirsch, T., Smith, J., \& Hirsch, R. (1991). An analysis of emergency department use by patients with minor illness. Annals of Emergency Medicine, 20(7), 743-748.

Simmons, B., Lanuza, D., Fonteyn, M., Hicks, F., \& Holm, K. (2003). Clinical Reasoning in Experienced Nurses. Western Journal of Nursing Research, 25(6), 701-719.

Simpson, E., \& Courtney, M. (2002). Critical thinking in nursing education: Literature review. International Journal of Nursing Practice, 8, 89-90.

Strauss, A., \& Corbin, J. (1998). Basics of qualitative research: Techniques and procedures for developing grounded theory, 2nd edn. Thousand Oaks, CA: Sage Publications.

Travers, D. A., Waller, A. E., Bowling, J. M., Flowers, D., \& Tintinalli, J. (2002). Five-level triage system more effective than three-level in tertiary emergency department. Journal of Emergency Nursing, 28(5), 395-400.

Ward A., \& Gracey J. (2006). Reflective practice in physiotherapy curricula: A survey of UK university based professional practice coordinators. Medical Teacher, 28, 32-39.

Washington, D. L., Stevens C. D., Shekelle, P. G., Baker, D. W., Fink, A., \& Brook, R. H (2000). Safely directing patients to appropriate levels of care: guideline-driven triage in the emergency service. Annals of Emergency Medicine, 36(1), 15-22.

Williams, R. M. (1996). Triage and emergency department services. Annals of Emergency Medicine, 27(4), 506-508.

Zimmermann, P. G. (2001). The case for a universal, valid, reliable 5-tier triage acuity scale for US emergency departments. Journal of Emergency Nursing, 27(3), 246-254. 